ISSN-PRINT 1794-9831 / E-ISSN 2322-7028

Vol. 18 No 2 / Mayo - Agosto, 2021 / Cúcuta, Colombia.

dot https://doi.org/10.22463/17949831.2849

Original

\title{
Bienestar espiritual de enfermeros de las unidades de cuidado intensivo en pandemia
}

\author{
Spiritual well-being of nurses at the intensive care unit during the pandemic \\ Bem-estar espiritual dos enfermeiros das unidades de terapia intensiva em pandemia
}

\author{
Silvia Liliana Ruiz-Roa ${ }^{1}$
}

\section{Resumen}

Objetivo: Determinar el bienestar espiritual y su relación con variables demográficas y profesionales en enfermeros que se desempeñan en Unidades de Cuidado Intensivo (UCI) durante la pandemia del COVID-19. Materiales y métodos: Estudio cuantitativo y transversal que determinó los niveles de bienestar espiritual mediante la escala de perspectiva espiritual (SPS) de Pamela Reed. La muestra se conformó con 116 enfermeros que trabajan en las UCI durante la emergencia sanitaria del COVID-19, en el periodo comprendido de junio a julio de 2020. La relación de las variables se determinó empleando el test de Fisher y las diferencias entre los grupos, utilizando Mann-Whitney y ANOVA. Fueron consideradas diferencias estadísticas significativas con $\mathrm{p}<0,05$. Resultados: Los participantes fueron predominantemente del sexo femenino, con edades inferiores a 45 años, estado civil solteros, con pregrado hasta nivel de formación. Se observaron elevados niveles de práctica, creencia y bienestar espiritual independientes de la situación de salud pública. Conclusión: La perspectiva espiritual varía según la edad, género, estado civil, formación académica e ingresos económicos. El alto bienestar espiritual en el enfermero que labora en la UCI durante la pandemia del COVID-19, es una fortaleza que puede apoyar el mantenimiento la salud mental, mejorando su capacidad de respuesta en escenarios de crisis.

Palabras clave: Espiritualidad; atención de enfermería; enfermeras y enfermeros; cuidado intensivo; COVID-19.

\footnotetext{
Abstract

Objective: Determine the spiritual well-being and its relationship with demographic variables of nursing professionals who work at the Intensive Care Unit (ICU) during the COVID-19 pandemic. Material and Methods: Quantitative, cross-sectional study that determined the levels of spiritual well-being through the Reed's Spiritual Perspective Scale (SPS) in 116 nurses that worked in the ICU during the public health emergency of COVID-19, from June to July of 2020. The relationship of the variables was determined using the Fisher test, and the differences between the groups using the Mann-Whitney $U$ test and the Analysis of Variance (ANOVA) test. Significant statistical differences, with $\mathrm{p}<0,05$, were considered. $\boldsymbol{R} \boldsymbol{e}$ sults: : The participants were predominantly female, under 45 years of age, single and with a bachelor's degree as their maximum level of education. High levels of practice, belief and spiritual well-being were observed regardless the public health situation. Conclusion: The spiritual perspective behaves differently depending on the age, gender, civil status, academic background, and income. A high well-being in the nursing professional who works at the ICU during the COVID-19 pandemic, is a strength that could help maintain their mental health,
}

\author{
Autor de correspondencia* \\ 1* Enfermera, M.Sc, Ph.D en Ciencias \\ Básicas B iomédicas. Profesora Auxil- \\ iar, Departamento de Atención Clínica y \\ Rehabilitación. Universidad Francisco \\ de Paula Santander. San José de Cúcuta, \\ Colombia. Correo: silvialilianarr@ufps. \\ edu.co (i) 0000-0002-4649-0944
}

Recibido: 9 febrero 2021

Aprobado: 13 abril 2021

Para citar este artículo / To reference this article / Para citar este artigo:

Ruiz-Roa SL. Bienestar espiritual de enfermeros de las un1dades de cuidado intensivo en pandemia. Rev. cienc. cuidad. $2021 ; \quad$ 18(2):8-18. https://doi org/10.22463/17949831.2849
(C) Universidad Francisco de Paula Santander. Este es un artículo bajo la licencia CC-BY-NC-ND 


\section{Scientific Journal of Nursing}

improving their capacity to respond to scenarios of crisis.

Palavras-chave: Spirituality; nurse care; nurses; intensive care; COVID-19.

\section{Resumo}

Objetivo: Determinar o bem-estar espiritual e a sua relação com as variáveis demográficas e profissionais dos enfermeiros que atuam nas Unidades de Terapia Intensiva (UTI) durante a pandemia da COVID-19. Materiais e métodos: Estudo quantitativo, transversal que determinou o bem-estar espiritual usando a escala de perspectiva espiritual (SPS) de Pamela Reed, em 116 enfermeiros que atuam em UTI durante a emergência sanitária da COVID-19 de junho a julho de 2020. A relação das variáveis estabeleceu-se com o teste de Fisher e as diferenças entre os grupos usando Mann-Whitney e ANOVA. Consideraram-se diferenças estatísticas significantes com $\mathrm{p}<0.05$. Resultados: Os participantes foram predominantemente do sexo feminino, com idade inferior aos 45 anos, solteiros e com a graduação como máximo nível de formação. Observaram-se altos níveis de prática crença e bem-estar espiritual, independente da situação de saúde pública. Conclusão: A perspectiva espiritual comporta-se de maneira diferenciada segundo a idade, sexo, estado civil, formação acadêmica e ingressos financeiros. O alto bem-estar espiritual no enfermeiro que trabalha na UTI durante a pandemia da COVID-19, é uma fortaleza que pode apoiar lhe na manutenção da sua saúde mental e melhorar a sua capacidade de afrontamento em cenários de crise.

Palavras-chave: Espiritualidade; cuidados de enfermagem; enfermeiras e enfermeiros; cuidados críticos; COVID-19

\section{Introducción}

La espiritualidad es la esencia de la naturaleza humana, es la motivación para la recuperación expresada por prácticas o creencias religiosas, que proporcionan paz interior, fortaleza y salud (1); además, articula el propósito del ser humano con la esperanza y la fe, trabajando de manera conjunta para producir beneficios para la salud en términos de prevención, recuperación de la enfermedad o hacer frente a la misma (2).

Antiguamente la espiritualidad era una dimensión humana estrechamente ligada a la experiencia religiosa; pese a lo anterior, en la actualidad la espiritualidad trasciende a todas las experiencias de la cotidianidad (3). Por tanto, es un elemento fundamental en situaciones en que las personas se perciben más vulnerables, ya sea por estar enfrentando una enfermedad como paciente o cuidador (4), incluso ante las situaciones de crisis y emergencia.

La espiritualidad es una dimensión humana bastante amplia, siendo complejos su abordaje y cuantificación; sin embargo, el establecimiento del bienestar espiritual es un aspecto que se aproxima fielmente al conocimiento de ésta dimensión (5).

Por su parte, el bienestar espiritual puede entenderse como el estado en que los sentimientos, comportam- ientos y condiciones cognitivas positivas, actúan favorablemente en la relación con sigo mismo, proporcionando al sujeto identidad, armonía interior y actitud positiva que contribuirá con su propósito de vida (6), favoreciendo su salud mental $(5,7)$ y trascendiendo a calidad de sus relaciones interpersonales y capacidad de afrontamiento.

La emergencia sanitaria mundial a consecuencia de la pandemia del virus SARS-CoV-2 (COVID-19) ha tenido un gran impacto sobre la humanidad. La población mundial ha experimentado crisis de miedo y ansiedad, afectando especialmente a aquellos que son mayores con afecciones de salud (8), sin dejar inmunes a la población joven y sana. También, preocupa de manera especial a los profesionales del sector salud quienes están en primera línea, enfrentando las consecuencias de esta enfermedad de progresión rápida y que ha generado grandes tasas de letalidad en el mundo, convirtiéndose en una importante causa de desequilibrio y estrés para los profesionales de enfermería.

Conviene subrayar que a pesar de la fortaleza que puede generar el bienestar espiritual para la salud física y mental de los enfermeros en su cotidianidad y en el cuidado a terceros, la escasa evidencia científica sobre el abordaje a profundidad de este aspecto es una debilidad. Al respecto se ha evidenciado que existe una necesidad urgente de su estudio, especialmente cuando 
la literatura ha demostrado que las prácticas y creencias espirituales están asociadas con un afrontamiento exitoso frente a situaciones estresantes (9). Este aspecto se debe tener muy en cuenta en los profesionales de enfermería quienes ejercen sus funciones en Unidades de Cuidado Intensivo (UCI) en periodos de pandemia, principalmente por su relación permanente con el sufrimiento de los pacientes internados y sus familiares (10). Adicionalmente, se debe agregar que las UCI son un escenario que requiere intervenciones asistenciales rápidas y oportunas ante situaciones de emergencia, con sobrecarga de trabajo, déficit de personal y condiciones de trabajo desfavorables, siendo fuentes estresoras permanentes (11); es decir, que son factores que pueden impactar sobre su capacidad de afrontamiento, generando alteraciones en el bienestar físico, mental y espiritual (12).

\section{Objetivos}

\section{Objetivo general}

Determinar el bienestar espiritual y su relación con variables demográficas y profesionales en enfermeros que se desempeñan en Unidades de Cuidado Intensivo (UCI) durante la pandemia de la COVID-19.

\section{Objetivos específicos}

- Describir las características sociodemográficas, académicas y profesionales de enfermeros que ejercen sus funciones en unidades de cuidado intensivo durante la pandemia.

- Establecer los niveles de práctica, creencia y bienestar espiritual de enfermeros que ejercen sus funciones en unidades de cuidado intensivo durante la pandemia.

- Identificar la diferencia de la práctica, creencia y bienestar espiritual según parámetros demográficos y profesionales de enfermeros que ejercen sus funciones en unidades de cuidado intensivo durante la pandemia.

- Establecer la relación existente entre la práctica, creencia y bienestar espiritual con las variables demográficas, académicas y profesionales de enfermeros que ejercen sus funciones en unidades de cuidado intensivo durante la pandemia.

\section{Materiales y Métodos}

Metodológicamente se llevó a cabo un estudio de tipo cuantitativo, descriptivo y transversal. La muestra fue calculada por muestreo no probabilístico y estuvo conformada por 116 enfermeros con edades comprendidas entre 23 y 52 años, siendo trabajadores asistenciales de las UCI de 10 instituciones de salud ( 5 de la ciudad de Bucaramanga y 5 de la ciudad de San José de Cúcuta, Colombia), durante el periodo inicial de la pandemia del COVID-19 comprendido entre abril y julio de 2020 . Los criterios de elegibilidad fueron: ser profesional de enfermería laborando en la UCI por un periodo ininterrumpido superior a 6 meses previos a la medición y su aceptación para participar en el estudio. Los datos fueron recolectados a través del autodiligenciamiento del cuestionario diseñado para la recolección de la información en la plataforma Google Forms ${ }^{\circledR}$.

Por otra parte, para medir la espiritualidad se utilizó la Escala de perspectiva espiritual (SPS por sus siglas en inglés) de Pamela Reed (13). Dicho instrumento está conformado por 10 ítems tipo Likert con 6 opciones de respuesta, y dos sub-escalas. La primera sub-escala pertenece a las prácticas espirituales y se mide con los ítems de 1 al 4, donde el 1 equivale a nunca, 2 menos de una vez al año, 3 más o menos una vez al año, 4 más o menos una vez al mes, 5 más o menos una vez a la semana, 6 más o menos una vez al día; con un puntaje máximo de 24 puntos. La interpretación general de la presente sub-escala sugerida por la investigadora se subdivide en: 1 a 8 baja práctica espiritual (alfa de Cronbach 0.8); 9-16 moderada práctica espiritual (alfa de Cronbach 0.91); y, 17- 24 puntos para una alta práctica espiritual (alfa de Cronbach 0.87).

La segunda sub-escala pertenece a las creencias espirituales con los ítems del 5 al 10, donde 1 extremadamente en desacuerdo, 2 en desacuerdo, 3 en desacuerdo más que en acuerdo, 4 de acuerdo más que en desacuerdo, 5 de acuerdo, 6 extremadamente de acuerdo; con un puntaje máximo de 36 puntos. La interpretación general de la presente sub-escala fue creada por la autora subdividiendo en: 1 a 12 baja creencia espiritual (alfa de Cronbach 0.79); 13-24 moderada creencia espiritual (alfa de Cronbach 0.82); 25-36 para una alta creencia espiritual (alfa de Cronbach 0.85), valores que se obtuvieron en el presente estudio .

La valoración global del bienestar espiritual mediante la aplicación de la escala SPS, se interpreta como: alto (46-60 puntos), moderado (31-45 puntos) y bajo (10-30 puntos), definidos previamente por Sierra \& Montalvo (10).

Del mismo modo, en la presente investigación se estableció la consistencia interna en las categorías de evaluación cualitativa creadas para las dos sub-escalas a través de Coeficiente de Confiabilidad de Aplha de Cronbach. La escala SPS fue utilizada previamente en 


\section{CienciagoCuidado}

Scientific Journal of Nursing

un estudio realizado en Colombia, en el que se consiguió un coeficiente de confiabilidad global para los 10 items de 0.8 (14), compatibles con el alfa de Cronbach calculado en el presente estudio de 0.82 .

Hay que mencionar además, que la presente investigación fue avalada por el comité de ética de la Universidad Francisco de Paula Santander (Acta de 06/2020). Se tuvo en cuenta la Resolución 8430 de 1993 (15), respecto a los principios éticos de beneficencia, privacidad, libertad de expresión y autonomía respaldado mediante el uso del consentimiento informado por escrito. Los datos que se obtuvieron fueron recolectados empleando Microsoft Excel $\AA$ y posteriormente analizados con el software estadístico Graph Pad Prism 5.0. Además, se utilizó estadística descriptiva a través de frecuencias absolutas y relativas. Para determinar las diferencias de los resultados obtenidos y en función de las mismas variables se empleó el test de Mann-Whitney y Kruskal-Wallis realizando el post-test de Dunn cuando fue pertinente.

De otro lado, para el análisis inferencial, se estableció la relación entre la espiritualidad y las variables categóricas: género, estado civil, formación académica y cargo laboral, a través del test exacto de Fisher, consideradas diferencias estadísticas significativas con valores $\mathrm{p}<0.05$.

\section{Resultados}

Fueron incluidos 116 enfermeros que ejercían sus labores en UCI durante el periodo de la pandemia del COVID-19, de los cuales el $67 \%$ son de la ciudad de San José de Cúcuta y 33\% están ubicados en instituciones del área metropolitana de Bucaramanga. En la Tabla 1 se observa que el $82,3 \%$ de estos profesionales tenían edades inferiores o iguales a los 45 años; el 78,5\% correspondían al género femenino y el $39,7 \%$ de estado civil soltero. La mayoría de los enfermeros manifestaron pertenecer a la religión católica $(76,7 \%)$ y cristiana $(14,7 \%)$. Además, se consideraron las variables relacionadas con su formación académica, remuneración económica y características de su cargo laboral, estableciéndose que el máximo nivel de formación académica alcanzado era el pregrado $(55,2 \%)$, con un tiempo de formación entre 1 a 5 años $(41,4 \%)$; con ingresos mensuales entre 471 y 700 USD (69\%); $\mathrm{y}$, desarrollo de funciones asistenciales $(91,4 \%)$ en las UCI durante el periodo de la emergencia sanitaria.

Tabla 1. Variables sociodemográficas, académicas y profesionales de enfermeros que ejercen sus funciones en unidades de cuidado intensivo de la ciudad de San José de Cúcuta y Bucaramanga durante la pandemia de la COVID-19.

\begin{tabular}{lrrc}
\hline & Variable & FA & FR (\%) \\
Edad (años) & $<25$ & 22 & 19 \\
Género & $25-45$ & 77 & 66,3 \\
Estado Civil & $>46$ & 17 & 14,7 \\
& $\mathrm{M}$ & 25 & 21,5 \\
& $\mathrm{~F}$ & 91 & 78,5 \\
& Soltero & 46 & 39,7 \\
Casado & 37 & 31,9 \\
Religión & 21 & 18,1 \\
& U.Libre/U. Estable & 11 & 9,5 \\
& Separado & 1 & 0,8 \\
& Viudo & 3 & 2,6 \\
Ateo & 89 & 76,7 \\
\hline Cristiano/Evangélico & 17 & 14,7 \\
& Otro & 7 & 6
\end{tabular}




\section{Scientific Journal of Nursing}

\begin{tabular}{lrcc}
$\begin{array}{l}\text { Máximo nivel } \\
\text { de formación } \\
\text { académica }\end{array}$ & Pregrado & 64 & 55,2 \\
& Especialización & 40 & 34,5 \\
& Maestría & 4 & 3,4 \\
$\begin{array}{l}\text { Tiempo desde su } \\
\text { formación (años) }\end{array}$ & Otro & 8 & 6,9 \\
& $1-5$ & 48 & 41,4 \\
& $6-10$ & 30 & 25,9 \\
& $11-15$ & 22 & 19 \\
$\begin{array}{l}\text { Ingresos mensu- } \\
\text { ales (USD) }\end{array}$ & $16-20$ & 11 & 9,5 \\
& $>21$ & 5 & 4,2 \\
& 4470 & 18 & 15,5 \\
& $471-700$ & 80 & 69 \\
\hline $\begin{array}{l}\text { Cargo desem- } \\
\text { peñado }\end{array}$ & $701-935$ & 17 & 14,7 \\
& $>936$ & 1 & 0,8 \\
\hline
\end{tabular}

$\mathrm{FA}=$ Frecuencia absoluta; $\mathrm{FR}=$ Frecuencia relativa; $\mathrm{USD}=$ Dólar americano

Fuente: Elaboración propia.

Para dar respuesta al objetivo de determinar el bie- que los enfermeros que se desempeñan en las nestar espiritual en la muestra estudiada, la Tab- UCI manifiestan una alta práctica (69\%), creenla 2 muestra los resultados obtenidos en la apli- cia $(87,1 \%)$ y bienestar espiritual $(71,6 \%)$. cación del instrumento SPS, demostrando

Tabla 2. Variables sociodemográficas, académicas y profesionales de enfermeros que ejercen sus funciones en unidades de cuidado intensivo de la ciudad de San José de Cúcuta y Bucaramanga durante la pandemia de la COVID-19.

\begin{tabular}{lccc}
\hline \multicolumn{1}{c}{ Variable $^{*}$} & FA & FR (\%) \\
\hline Práctica espiritual $^{(*)}$ & Baja & 4 & 3,5 \\
& Moderada & 32 & 27,5 \\
& Alta & 80 & 69 \\
Creencia espiritual $^{(*)}$ & Baja & 3 & 2,6 \\
& Moderada & 12 & 10,3 \\
& Alta & 101 & 87,1 \\
\hline Bienestar espiritual & Bajo & 7 & 6 \\
& Moderado & 26 & 22,4 \\
& Alto & 83 & 71,6 \\
\hline
\end{tabular}

$(*)=$ Categorías creadas para indicar cómo fue descrito en la metodología; FA= Frecuencia absoluta; $\mathrm{FR}=$ Frecuencia relativa

Fuente: Elaboración propia. 


\section{CienciageCuidado}

\section{Scientific Journal of Nursing}

Con el propósito de identificar la diferencia en la práctica, creencia y bienestar espiritual dentro de las variables demográficas y profesionales, se aplicó el test de Mann-Whitney y Kruskall-Wallis, como se muestra en las Figuras 1 y 2, observándose menores prácticas espirituales en los enfermeros con edades inferiores a los 25 años $(p<0,01)$, del género masculino $(p=0,04)$, estado civil soltero $(p=0,03)$ y que manifestaron ser ateos $(p<0,01)$. Esta misma tendencia se aceptuó en los enfermeros cuyo mayor grado de formación académi-

Figura 1. Comparación de las puntuaciones obtenidas en las dimensiones práctica, creencia y bienestar espiritual según las variables sociodemográficas de enfermeros que ejercen sus funciones en unidades de cuidado intensivo de la ciudad de Cúcuta y Bucaramanga durante la pandemia del COVID-19.

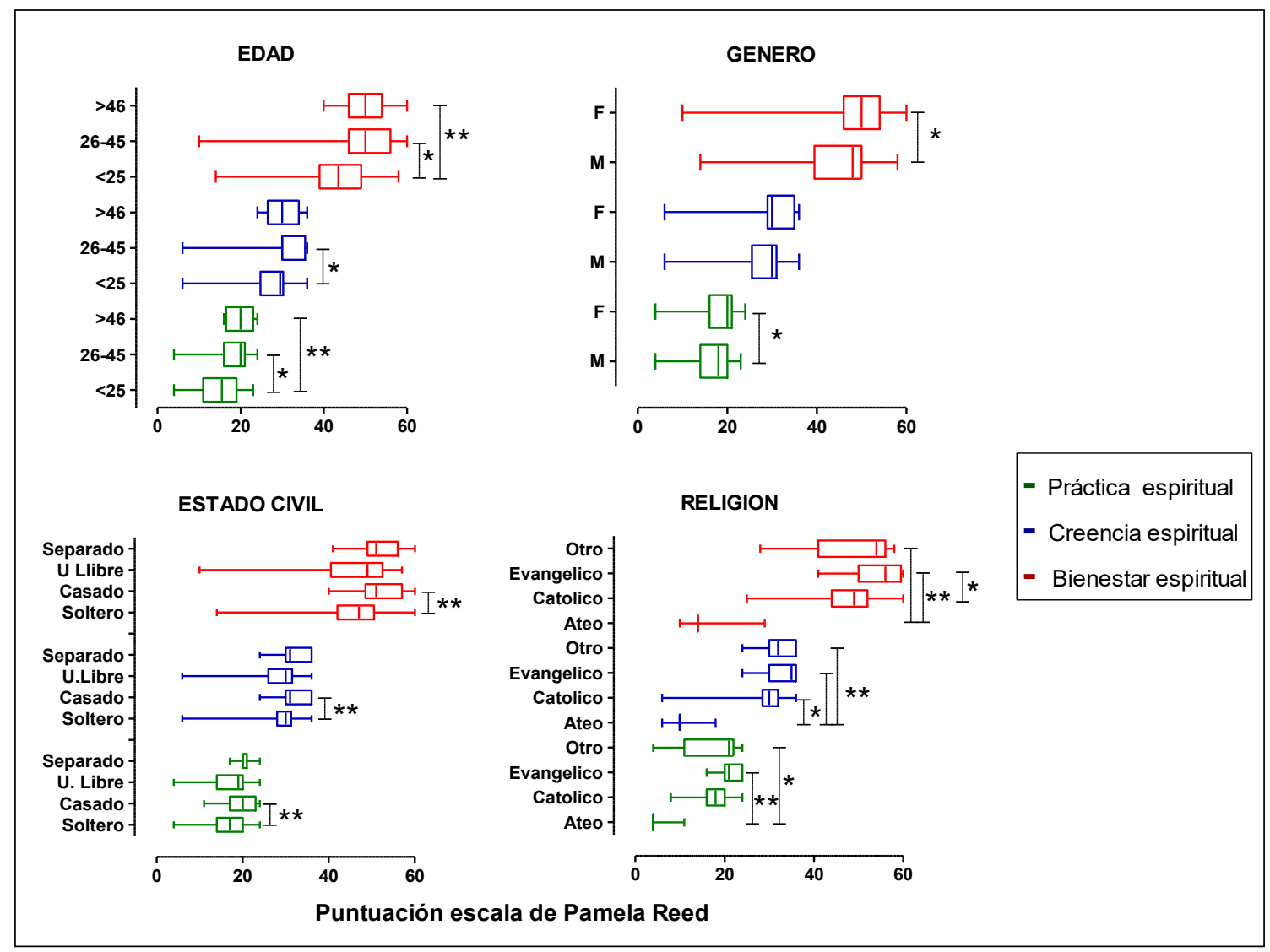

$(*)$ valores $\mathrm{p}<0.05 ;(* *)$ valores $\mathrm{p}<0.01$.

Fuente: Elaboración propia. 


\section{Scientific Journal of Nursing}

Figura 2. Comparación de las puntuaciones obtenidas en las dimensiones práctica, creencia y bienestar espiritual según las variables académicas y profesionales de enfermeros que ejercen sus funciones en unidades de cuidado intensivo de la ciudad de Cúcuta y Bucaramanga durante la pandemia del COVID-19.

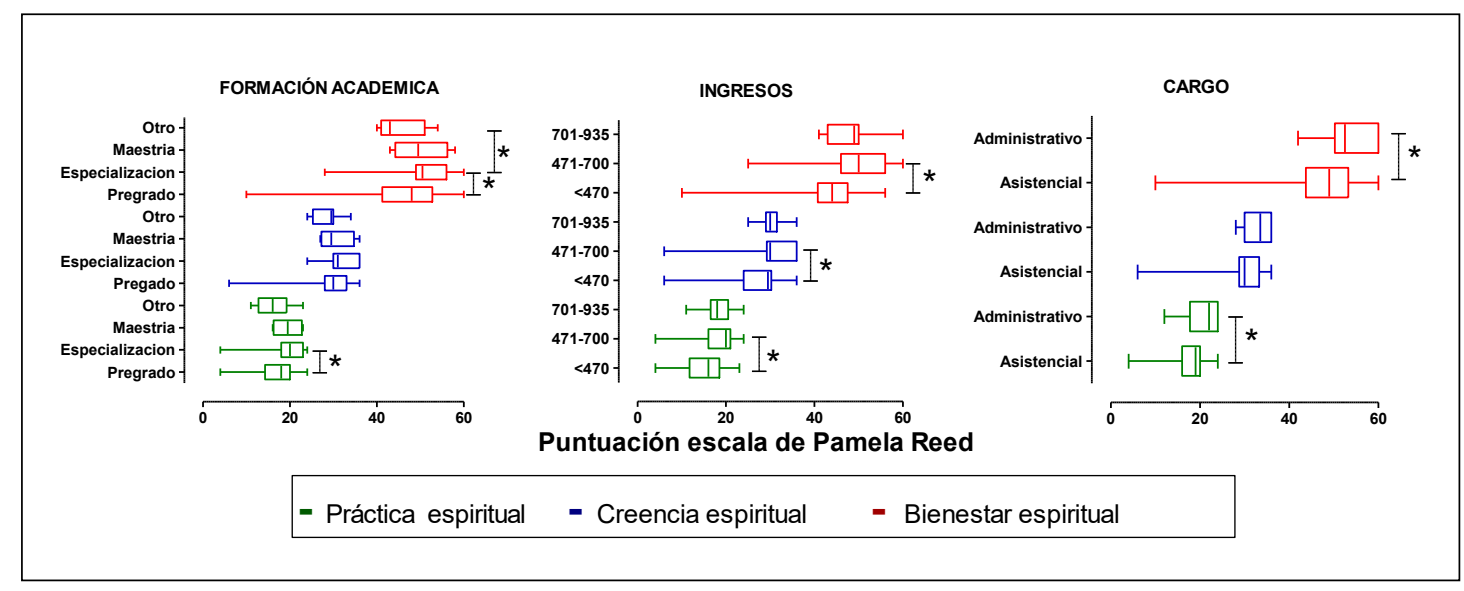

$(*)$ valores $\mathrm{p}<0.05$

Fuente: Elaboración propia.

Para establecer la relación existente entre la práctica, la creencia y el bienestar espiritual, con las variables sociodemográficas, las académicas y las profesionales de los enfermeros que ejercen en las UCI, la Tabla 3 muestra que ser ateo está relacionado con menor práctica $(p<0,01)$, creencia $(p<0,01)$ y bienestar espiritual $(p<0,01)$. Por otro lado, ser enfermero del sexo femenino y tener ingresos mensuales superiores a los 471USD está relacionado con mayores niveles de práctica, creencia y bienestar espiritual.

Tabla 3. Relación estadística de las variables sociodemográficas, académicas y profesionales con la práctica, creencia y bienestar espiritual de enfermeros que ejercen sus funciones en unidades de cuidado intensivo de la ciudad de Cúcuta y Bucaramanga durante la pandemia del COVID-19.

\begin{tabular}{lccc}
\hline \multicolumn{1}{c}{ Variable } & Práctica Espiritual & Creencia espiritual & Bienestar espiritual \\
\hline Edad & $(\mathrm{p})$ & $(\mathrm{p})$ & $(\mathrm{p})$ \\
\hline Género & $\mathrm{NS}$ & 0,08 & $\mathrm{NS}$ \\
\hline Estado Civil & $0,02^{*}$ & $\mathrm{NS}$ & $0,0004^{*}$ \\
\hline Religión & $\mathrm{NS}$ & $\mathrm{NS}$ & $\mathrm{NS}$ \\
\hline Formación académica & $0,001^{*}$ & $0,0006^{*}$ & $0,0004^{*}$ \\
\hline Ingresos mensuales & $\mathrm{NS}$ & $\mathrm{NS}$ & $\mathrm{NS}$ \\
Cargo & 0,07 & $0,04^{*}$ & $0,02^{*}$ \\
\hline
\end{tabular}

$(\mathrm{p})=$ Valores $\mathrm{p}$ obtenidos; $\mathrm{NS}=$ sin significancia estadística; $(*)$ relación evaluada mediante la aplicación del Test exacto de Fisher.

Fuente: Elaboración propia. 


\section{Discusión}

De acuerdo con el presente estudio se determinó el perfil de las variables práctica, creencia y bienestar espiritual, en enfermeros que actúan en las UCI durante el periodo de la pandemia del COVID-19. A pesar de que la emergencia sanitaria tiene un impacto sin precedentes en la salud pública del milenio, las características sociodemográficas de la muestra conformada por los enfermeros sujeto de estudio no son distantes a los de enfermeros y cuidadores cuya espiritualidad ha sido estudiada previamente a nivel nacional y en países del primer mundo.

Un estudio previo realizado por Torskenæs et al. (16) estableció la diferencia del concepto de espiritualidad en enfermeros y cuidadores de Malta y Noruega, concluyendo que la mayoría de los cuidadores tenían edades inferiores a los 50 años; sin embargo, su estado civil era casados y se consideraban moderadamente religiosos (16) destacándose que la religión más practicada por las poblaciones de estos países es la católica/ cristiana (17), tal como ocurre en Colombia (18). Estas variables evidenciaron datos semejantes en un estudio realizado con enfermeras que se desempeñan en UCI de un hospital público de Cincinnati (19), así como en uno aplicado al equipo de salud de las UCI Neonatal de hospitales infantiles de las ciudades de Pennsylvania y Philadelfia (20), donde la mayoría de estos equipos estaban integrados predominantemente por enfermeras del género femenino, con edades inferiores a los 45 años y pertenecientes a la religión católica/cristiana.

En el escenario nacional, el bienestar espiritual ha sido determinado en estudiantes de enfermería (21) y en enfermeros que trabajan en UCI del litoral caribe (10); éste último estudio demostró que los resultados se hallaron en sujetos predominantemente del género femenino, con edad promedio de 33.2 años, estado civil casado y pertenecientes a la religión católica/cristiana. Igualmente indicó que los ingresos mensuales no exceden los 720 USD, tal como fue establecido en la presente investigación.

Enfatizando sobre la formación académica de los enfermeros que ejercen en las UCI, la literatura nacional (10) como la internacional (19), han demostrado que su máximo nivel de formación es el pregrado, con poca frecuencia las enfermeras están especializadas o con formación en postgrado en los servicios de terapia intensiva.

La SPS fue el instrumentó elegido para determinar el bienestar espiritual de los enfermeros evaluados en la presente investigación, dando prioridad al abordaje del concepto de espiritualidad como una experiencia del ser humano, particularmente relevante en situaciones donde la conciencia de la muerte se hace más evidente (22), como es el caso de la actual pandemia. Fueron identificadas diferencias con respecto a las variables práctica, creencia y bienestar espiritual según la edad, género, estado civil, religión, nivel de formación académica, ingresos económicos y el cargo desempeñado por los enfermeros que laboran dentro de las UCI. Además, se enfatizó en el valor de ésta información, dado que no se encuentran antecedentes en la literatura nacional e internacional que establezcan diferencias en la práctica, creencia y bienestar espiritual, según las diferentes variables demográficas y académicas de enfermeros y cuidadores.

Es importante resaltar que de forma innovadora, el presente estudio estratificó los resultados de las sub-escalas sobre práctica y creencia espiritual sugeridas para la valoración global empleadas en la literatura, creando categorías cualitativas que permitieron evaluar éstas variables. Igualmente, se compararon las observaciones con los antecedentes, realizándose la estratificación de los resultados en un estudio que empleó el SPS en el territorio nacional (10). De igual forma se obtuvieron hallazgos que sirvieron para demostrar que entre los enfermeros que actúan en las UCI en el territorio colombiano, existe una alta incidencia en las variables relacionadas con: prácticas, creencias y bienestar espiritual, independientes de la situación de salud pública por la presencia del COVID-19.

En el ámbito internacional altos niveles de bienestar espiritual en enfermeros han sido ampliamente descritos $(23,24)$. Recientemente Ricci-Allegra (25) demostró que los enfermeros que trabajan en cuidados paliativos tienen altos niveles de bienestar espiritual. De igual forma demostró que estas actitudes son estadísticamente superiores en quienes tienen algún tipo de filiación religiosa comparados con los enfermeros que no la tienen, tal como se prueba en este trabajo.

Así mismo, fuentes internacionales han descrito la relación entre el tiempo de la experiencia profesional y la ausencia de relación con otras variables demográficas, la práctica espiritual y el cuidado espiritual en enfermería $(26,27)$. En cambio con este estudio se demostró que ser enfermero del género femenino, con algún tipo de filiación religiosa y disponer de ingresos mensuales superiores a los 471 USD, son factores que influyen en los niveles de bienestar espiritual. Es decir, que a pesar de las diferencias demográficas y profesionales de los sujetos estudiados, la práctica, la creencia y el bienestar espiritual son variables independientes de la edad, el estado civil, el nivel de formación académica y el tipo 
de cargo de las enfermeras que trabajan en las UCI en el periodo de emergencia sanitaria. Fenómeno que se evidencia en esta investigación, aún a costa de las limitaciones.

Es necesario recalcar que se deben conocer las condiciones de bienestar espiritual que tienen los enfermeros, debido a la relación que existe entre esta variable y la actitud que acompaña sus acciones de cuidado espiritual, así como su grado de compromiso profesional (28), que en situaciones de crisis es determinante. Por otro lado, se ha demostrado que la fortaleza espiritual es un factor protector positivo frente a la competencia inmunológica, reduciendo las tasas de infección, lo que facilita la aceptación de los tratamientos para las infecciones (29) como son: el aislamiento social, las medidas de higiene y el autocuidado para reducir la propagación del COVID-19.

También, se ha demostrado que las situaciones de emergencia incrementan el estrés en el escenario laboral, la ansiedad y la depresión (30), afectando la calidad y la eficiencia de los cuidados del enfermero que actúa en UCI (19). En consecuencia se debe contar con el respaldo institucional que incluya la preparación frente a situaciones de emergencia sanitaria; igualmente, hay que abordar las necesidades holísticas de los enfermeros, incluyendo la atención de su dimensión espiritual, de tal forma que se contribuya a la generación de mayores fortalezas para la atención de la salud en situaciones de crisis.

\section{Conclusiones}

- Los enfermeros que actúan en las UCI de San José de Cúcuta y Bucaramanga que han enfrentado la pandemia del COVID-19, son generalmente profesionales exclusivamente con formación de pregrado, que cuentan con altos niveles de práctica, creencia y bienestar espiritual, independiente de la emergencia de salud pública.

- El presente estudio de forma innovadora concluye que las variables que componen la valoración de la perspectiva espiritual, se comportan de forma diferenciada según la edad, el género, el estado civil, la religión, el grado de formación académica y los ingresos económicos mensuales de los enfermeros que trabajan en UCI; así mismo, que algunas de éstas condiciones (género, religión e ingresos mensuales), están relacionadas con mayores niveles de bienestar espiritual.

- Ser pioneros en el abordaje y análisis de la perspectiva espiritual de los enfermeros que están en la línea de frente de una emergencia en salud pública, pone en evidencia la necesidad de incluir estrategias sobre el fortalecimiento del bienestar espiritual en la educación superior de futuros enfermeros. Propuesta que se fundamenta en el hecho de que la mayoría de los profesionales a cargo de los servicios asistenciales de cuidado intensivo, tienen como máximo nivel de formación académica el pregrado.

Agradecimientos: a la profesora Sandra Milena Martínez Rojas, Coordinadora del curso Cuidado del Adulto II del programa de enfermería UFPS, por el trabajo colaborativo y apoyo logístico.

Limitaciones: La autora declara que, para enriquecer la discusión de los resultados, fueron empleados artículos científicos de estudios realizados en poblaciones con características y condiciones semejantes, considerando algunas referencias con un periodo de publicación superior a los 5 años.

Conflicto de Intereses: La autora declara no tener ningún conflicto de interés.

\section{Referencias Bibliográficas}

1. Blasdell ND. The Evolution of Spirituality in the Nursing Literature. International Journal of Caring Sciences [Internet], 2015 [Consultado 08/2020]; 8(3):756-764. Disponible en: http://internationaljournalofcaringsciences.org/docs/27 Basdell_special_8 3.pdf

2. Coyle J. Spirituality and health: towards a framework for exploring the relationship between spirituality and health. Journal of Advanced Nursing, 2002; 37(6), 589-597. http://doi.org/10.1046/j.1365-2648.2002.02133.x

3. Soto AM, Olivella MC, Bastidas CV. Cuidado espiritual al adulto mayor, elemento del conocimiento y práctica de enfermería. Revista Ciencia y Cuidado, 2020; 17(1):123-131. https://doi.org/10.22463/17949831.1563

4. Rogers M, Wattis J. Spirituality in nursing practice. Nursing Standard, 2015; 29(39), 51-57. http://doi. org/10.7748/ns.29.39.51.e9726 
Scientific Journal of Nursing

5. Volcan SMR, Souza PLR, Mari JJ, Horta BL. Relationship between spiritual well-being and minor psychiatric disorders: a cross-sectional study. Revista de Saúde Pública, 2003; XXXVII (4):440-5. http://doi.org/10.1590/ $\underline{\text { S0034-89102003000400008 }}$

6. Velasco L, Rioux L. The Spiritual Well-Being of Elderly People: A Study of a French Sample. Journal of Religion and Health, 2014; 53(4):1123-37. http://doi.org/10.1007/s10943-013-9710-5

7. George S, McDonnell M, Diiorio C, Laderman G. Spiritual well-being, depressive symptoms, and immune status among women living with HIV/AIDS. Women Health, 2009; XLIX(2-3):119-43. http://doi. org/10.1080/03630240902915036

8. Koenig HG. Ways of protecting religious older adults from the consequences of COVID-19. American Journal of Geriatric Psychiatry, 2020; 28(7), 776-779. http://doi.org/10.1016/j.jagp.2020.04.004

9. Koenig G. Religion, spirituality and psychiatry: a new era in mental health care. Revista de Psiquiatria Clínica, 2007; XXXIV (supl 1):5-7. http://doi.org/10.1590/S0101-60832007000700002

10. Sierra L, Montalvo A. Bienestar espiritual de enfermeras y enfermeros en unidades de cuidado intensivo. Avances en Enfermería [Internet], 2012 [Consultado en 08/2020]; XXX (1):64-74. Disponible en: http://www. scielo.org.co/pdf/aven/v30n1/v30n1a07.pdf

11. Lima MB, Silva LM, Monteiro FC, Martins RA, Matos HH. Agentes estressores em trabalhadores de enfermagem com dupla ou mais jornada de trabalho. Revista de Pesquisa Cuidado é Fundamental Online [Internet], 2013 [Consultado en 08/2020]; V (1):3259-3266. Disponible en: https://www.redalyc.org/pdf/5057/505750897010. pdf

12. Jofré V, Valenzuela S. Burnout en personal de enfermería de la Unidad de Cuidados Intensivos Pediátricos. Aquichan [Internet], 2005 [Consultado el 08/2020]; 5(1):56-63. Disponible en: http://www.scielo.org.co/pdf/ aqui/v5n1/v5n1a06.pdf

13. Reed PG. An Emerging paradigm for the investigation of spirituality in nursing. Research in Nursing \& Health, 1992; 15 (1):349-357. http://doi.org/10.1002/nur.4770150505

14. Jaramillo D, Ospina D, Cabarcas G, Humpreys J. Resiliencia, espiritualidad, aflicción y tácticas de resolución de conflictos en mujeres maltratadas. Revista Salud Publica [Internet], 2005 [Consultado el 08/2020]; 7(3):28192. Disponible en: http://www.scielo.org.co/pdf/rsap/v7n3/v7n3a04.pdf

15. Ministerio de Salud. Resolución $\mathrm{N}^{\mathrm{o}}$ 008430. Por la cual se establecen las normas científicas, técnicas y administrativas para la investigación en salud. Bogotá: Ministerio de Salud; 1993 [Consultado en 08/2020]. p. 1- 12. Disponible en: https://www.hospitalsanpedro.org/images/Comite_Investigacion/Resolucion 8430 de 1993. pdf

16. Torskenæs KB, Baldacchino DR, Kalfoss M, Baldacchino T, Borg J, et al. Nurses' and caregivers' definition of spirituality from the Christian perspective: a comparative study between Malta and Norway. Journal of Nursing Management, 2015; 23(1):39-53. http://doi.org/10.1111/jonm.12080

17. Gouder A. Roman Catholic Religious Affiliation Regis-ter. Archbishop's Curia, 2010; Floriana, Malta.

18. Bidegain AM, Demera JD. Libro Globalización y diversidad religiosa en Colombia. Colombia. 2005; Editorial Universidad Nacional de Colombia.

19. Campbell D. Spirituality, Stress, and Retention of Nurses in Critical Care. Dimensions of critical care nursing, 2013; 23(2):78-83. http://doi.org/10.1097/DCC.0b013e31828083a4 
Scientific Journal of Nursing

20. Coughlin K, Mackley A, Kwadu R, Shanks V, Sturtz W, et al. Characterization of Spirituality in MaternalChild Caregivers. Journal of Palliative Medicine, 2017; 20(9):994-997. http://doi.org/10.1089/jpm.2016.0361

21. Fajardo-Ramos E, Henao-Castaño AM. Bienestar espiritual de los estudiantes de enfermeria. Cultura de los cuidados, 2019; 23 (54):23-31. http://doi.org/http://dx.doi.org/10.14198/cuid.2019.54.03

22. Canaval G., Gonzáles MC, Sánchez MO. Espiritualidad y resiliencia en mujeres maltratadas que enuncian su situación de violencia de pareja. Revista Colombia Médica [Internet], 2007 [Consultado en 08/2020]; 38(4) (supl 2):72-78. Disponible en: http://www.scielo.org.co/pdf/cm/v38n4s2/v38n4s2a8.pdf

23. Tuck I, Pullem L, Wallace D. A Comparative Study of the Spiritual Perspectives and Interventions of Mental Health and Parish. Issues in Mental Health Nursing, 2001; 22(6):593-605. http://doi.org/10.1080/0161284017 $\underline{50364138}$

24. Conner N, Eller L. Spiritual perspectives, needs and nursing interventions of Christian African-Americans. Journal of Advanced Nursing, 2004; 46(6):624-32. http://doi.org/10.1111/j.1365-2648.2004.03053.x

25. Ricci-Allegra P. Spiritual Perspective, Mindfulness, and Spiritual Care Practice of Hospice and Palliative Nurses. Journal of Hospice \& Palliative Nursing, 2018; 20(2):172-179. http://doi.org/\%2010.1097/ $\underline{\text { NJH.0000000000000426 }}$

26. Mamier I. Nurses Spiritual Care Practices: Assessment Type, Frequency, and Correlates. Tesis de doctorado no publicada [Internet]. School of Nursing, Loma Linda University. 2009 [Consultada en 08/2020]; Recuperado de: https://scholarsrepository.llu.edu/cgi/viewcontent.cgi?referer=https://scholar.google.com.co/\&httpsre$\underline{\text { dir }=1 \& \text { article }=1488 \& \text { context }=\text { etd }}$

27. Ronaldson S, Hayes L, Aggar C, Green J, Carey M. Spirituality and spiritual caring: nurses perspectives and practice in palliative and acute care environments. Journal of Clinical Nursing, 2012; 21(15-16), 2126-2135. http://doi.org/10.1111/j.1365-2702.2012.04180.x

28. Chiang YC, Lee HC, Chu TL, Han CY, Hsiao YC. The impact of nurses' spiritual health on their attitudes toward spiritual care, Professional commitment, and caring. Nursing Outlook, 2016; 64(3), 215-224. http://doi. org/0.1016/j.outlook.2015.11.012

29. Oman D, Riley LW. Why infectious Diseases, Religion, and Spirituality. In: Oman D. (eds) Why Religion and Spirituality Matter for Public Health. Religion, Spirituality and Health: A Social Scientific Approach, 2018; (153-163). Springer, Cham. https://doi.org/10.1007/978-3-319-73966-3 9

30. Chen R, Chou K-R, Huang Y-J, Wang T-S, Liu S-Y, Ho L-Y. Effects of a SARS prevention programme in Taiwan on nursing staff's anxiety, depression and sleep quality: a longitudinal survey. International journal of nursing studies. 2006;43(2):215-25. https://doi.org/10.1016/j.ijnurstu.2005.03.006 